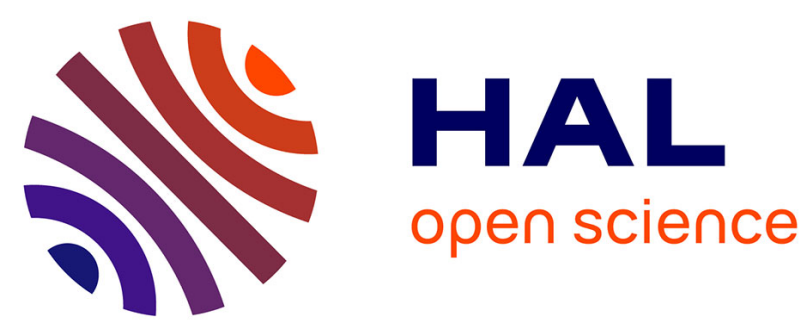

\title{
Le marketing de la collecte de legs: de la légitimité à proposer l'immortalité à la gestion relationnelle
}

Sophie Rieunier, Géraldine Michel

\section{To cite this version:}

Sophie Rieunier, Géraldine Michel. Le marketing de la collecte de legs: de la légitimité à proposer l'immortalité à la gestion relationnelle. Décisions Marketing, 2013, 69, pp.61-75. 10.7193/dm.069.61.75 . hal-02050138

\section{HAL Id: hal-02050138 https://hal.science/hal-02050138}

Submitted on 9 Mar 2019

HAL is a multi-disciplinary open access archive for the deposit and dissemination of scientific research documents, whether they are published or not. The documents may come from teaching and research institutions in France or abroad, or from public or private research centers.
L'archive ouverte pluridisciplinaire HAL, est destinée au dépôt et à la diffusion de documents scientifiques de niveau recherche, publiés ou non, émanant des établissements d'enseignement et de recherche français ou étrangers, des laboratoires publics ou privés. 
Revue Décisions Marketing

2013, N69, janvier - mars, $61-75$

Le marketing de la collecte de legs :

de la légitimité à proposer l'immortalité à la gestion relationnelle

\author{
Sophie Rieunier \\ Maître de conférences \\ IAE de Paris - GREGOR - 21 rue Broca - 75005 Paris \\ Mail : rieunier.iae@univ-paris1.fr - \\ Géraldine Michel \\ Professeur des Universités \\ IAE de Paris - GREGOR - 21 rue Broca - 75005 Paris \\ Mail : michel.iae@univ-paris1.fr -
}

\title{
Remerciements
}

Nous souhaitons remercier l'ensemble des 24 managers qui ont accepté d'être interrogées pour cette recherche :

1. Alexis Vandevivère (Adfinitas Paris),

2. François Rubio (Médecins du Monde),

3. Jean-Marie Destrée (Secours Catholique),

4. Isabelle Bourgoin (France Générosité)

5. Eléonore Valais de Sibert (Musée du Louvre)

6. Scott Hunt et Emmanuelle Poupeau (Lycée Français de New York)

7. Donna Thomas (China Institute in America)

8. Catherine Desjacques et Elisabetta Scanferla Schmitt (ESCP)

9. Arthur Moore (Consultant en fundraising - New York)

10. Frédéric Grosjean (Institut Pasteur)

11. Frédéric Fournier (Optimus)

12. Catherine Vialle (Ernst et Young)

13. Daniel Bruneau (les petits frères des Pauvres)

14. Anne-Marie Schmit et Florence Teissier (Greenpeace)

15. Serena Gavazzi (Château de Versailles)

16. Jean-François Riffaud (Croix Rouge Française)

17. Priya Roychowdhury (Muséum d'histoire naturelle de New York)

18. Florence Mahé Dombis (Muséum d'histoire naturelle de Paris)

19. Franck Hourdeau (MédiaprismGroup)

20. Barbara de Colombe (HEC Paris)

21. Anne Fleury (Diocèse de Paris - Eglise de France) 


\title{
Le marketing de la collecte de legs :
}

\section{de la légitimité à proposer l’immortalité à la gestion relationnelle}

\section{Résumé}

Associations de solidarité, établissements d'enseignement supérieurs et institutions culturelles ont de plus en plus de besoins financiers et de moins en moins de subventions publiques. La collecte de fonds devient alors fondamentale pour leur survie. Cet article a pour objectif d'identifier les leviers de la collecte de legs au travers d'une étude qualitative réalisée auprès de 21 professionnels de la collecte. Les résultats montrent le rôle déterminant de la légitimité à « offrir de l'immortalité » et l'importance de la relation nouée entre l'organisation et le testateur potentiel. Enfin, les implications organisationnelles et éthiques de la collecte de legs sont identifiées.

Mots clés : donateurs - don - legs - marketing relationnel - collecte de fonds

\section{The legacy marketing: from the legitimacy of "immortality offer" to the relationship management}

\begin{abstract}
Charities, universities and cultural institutions have greater and greater financial need and less and less public funding. Fundraising has thus become fundamental to their survival. The purpose of this article is to analyse the marketing of bequests through "face to face” interviews with fundraisers specialized in planned giving. In particular, this research shows the determining roles of legitimacy to "propose immortality" and the importance of the relationship formed between the organisation and the potential donor. Finally, the organisational and ethical implications of bequests are identified.
\end{abstract}

\section{Keywords}

Bequest - Giving - Legacy - Customer Relationship Management - Fundraising. 


\section{Introduction}

Le comportement de don est inhérent à toutes les sociétés et se manifeste dès le début de la vie au travers du don fait par les parents aux enfants; sans don, le nouveau né ne pourrait pas survivre bien longtemps et il ne pourrait y avoir d'éducation (Bergadàa et al., 2011). De nombreux sociologues, philosophes, économistes et anthropologues se sont intéressés au don. Parmi eux, c’est sans doute Mauss (1954) qui reste le plus cité au travers de sa théorie du « don contre don » analysée dans le fonctionnement des sociétés primitives. En France, aujourd'hui, nous pouvons distinguer deux formes de dons : le don fait à ses proches et le don fait à des anonymes (et notamment, aux associations). C’est ce deuxième type de don qui anime la réflexion menée dans cet article (encadré 1).

\section{Encadré 1 - Les différentes modalités de dons à des anonymes}

Il existe différentes formes de dons pour venir en aide aux anonymes. Elles peuvent être structurées selon l'implication financière, temporelle et physique demandée au donateur :

- le don de vêtements (peu impliquant sur les trois dimensions),

- le don manuel (assez peu impliquant sur les trois dimensions),

- le don en nourriture (un peu plus impliquant financièrement),

- le don en chèque (plus impliquant financièrement),

- le don par prélèvement automatique qui témoigne d'un engagement sur une longue durée (encore plus impliquant financièrement et de manière temporelle),

- le don de sang ou d'organes qui est lié à une implication physique forte,

- le don de temps (implication temporelle forte),

- la donation (qui doit se faire devant notaire, du vivant du donateur et qui est très impliquante financièrement),

- $\quad$ et enfin, le don « ultime » souvent majeur, très impliquant pour l'individu : le legs.

Faire un legs revient à donner à sa mort au travers de son testament, tout ou partie de son patrimoine (meubles, objets, patrimoine financier, assurances vies, immobilier, droits d'auteurs). Selon un rapport d'Ernst et Young, les legs représentaient en 2007, 34\% des ressources privées des associations et fondations (Dutheil et Vialle, 2009). Le potentiel de collecte de legs est très élevé puisque les legs aux associations et fondations, seraient aujourd'hui d'environ 1 milliard d'euros alors que leur potentiel est évalué à 9 milliards (Destrée, 2009). D’ailleurs, « selon l'analyse des comptes emploi - ressources des associations et fondations, les libéralités se sont accrues de 70\% sur ces 5 dernières années » (Dutheil et Vialle, 2009). La totalité des successions en France s’élève à un montant de 60 milliards d'euros pour 2006. Il y a donc une très grande marge de développement de la collecte sur ce domaine des legs. Cependant, encore très peu d'organisations mettent en place une réelle stratégie marketing de collecte de legs. La plupart reçoivent de temps en temps des legs, mais sans réellement l'avoir demandé. Or, 
la collecte de legs fait appel à une démarche volontaire de la part de l'organisation collectrice qui se doit d'être professionnelle vis-à-vis du testateur si elle souhaite réellement développer cet axe de collecte.

L’objectif de cet article est de mettre en évidence les leviers de la collecte de legs. Pour cela nous avons mis en perspective les recherches antérieures, puis nous avons mené 21 entretiens auprès de spécialistes du legs (encadré 2) issus de plusieurs types d'organisations: les associations de solidarité, l'enseignement supérieur et les organisations culturelles. Les contributions majeures de cet article reposent sur trois aspects : d'une part, une synthèse de la littérature sur les motivations et les freins des individus face au legs (partie I), d'autre part une identification des leviers en termes « d'offre », de communication et de marketing relationnel sur lesquels les organisations peuvent jouer pour la collecte de legs (partie II), enfin, une mise en évidence des changements organisationnels engendrés par cette collecte.

\section{Encadré 2 - Méthodologie de la recherche}

L'objectif de cette recherche est de comprendre la démarche marketing utilisée par les organisations pour collecter les legs. Par conséquent, cette recherche repose sur une démarche qualitative réalisée autour de 21 entretiens auprès de professionnels de cette collecte.

- Quinze entretiens ont été réalisés auprès de directeurs de la collecte de fonds dans trois milieux: d'une part, les associations et fondations de solidarité (association diocésaine de Paris, Croix Rouge Française, Greenpeace, Institut Pasteur, petits frères des Pauvres, Secours Catholique, Médecins du Monde), d'autre part, l'enseignement supérieur (ESCP, HEC Paris, Lycée Français de New York, Muséum d’histoires naturelles de Paris ${ }^{1}$ ), enfin, le secteur culturel (château de Versailles, China Institute de New York, muséum d'histoires naturelles de New York, musée du Louvre).

- Quatre autres entretiens ont été conduits auprès d'agences spécialisées dans la collecte de fonds : Arthur Moore Consultant (New York), Adfinitas Paris, Optimus, Médiaprism.

- Enfin, deux autres structures ont été rencontrées pour leur expertise sur le domaine du legs : France Générosité et Ernst \& Young.

Nous nous sommes intéressés aux organisations actives dans la collecte de legs ou qui ont une démarche originale sur ce thème. Les entretiens, de type semi-directifs (guide d'entretien en annexe 1) se sont déroulés sur le lieu de travail des différentes personnes interrogées. Ils ont duré entre 1 et 2 heures. Ils n’ont pas été enregistrés afin d'assurer la liberté des propos des individus. Les notes, prises durant tout l'entretien, ont été retranscrites, puis validées par les interviewés. Une analyse de contenu thématique manuelle a ensuite été menée.

\footnotetext{
${ }^{1}$ Le Muséum d'histoire naturelle n'est pas un établissement culturel puisqu’il dépend du ministère de l'environnement et du ministère de l'enseignement supérieur et de la recherche. Il a le statut de Grand établissement.
} 


\section{Les motivations et freins au legs : synthèse de la littérature}

\section{Les motivations au legs}

Il existe très peu de littérature sur le comportement de legs aux ONG. Les rares travaux publiés sur ce sujet sont basés sur des données anglaises et américaines. Ils ont identifié sept motivations au legs :

1. le fait de ne pas avoir d'enfants à qui transmettre son patrimoine (James, 2009 ; Sargeant, Wymer et Hilton, 2006).

2. la générativité : l'immortalité symbolique de son nom (Sargeant et Hilton, 2005 ; Sargeant et Shang, 2008, Sargeant, Wymer et Hilton, 2006), l'immortalité symbolique de son histoire personnelle (Sargeant, Wymer et Hilton, 2006), l'envie de transmettre aux générations futures (Sargeant, Wymer et Hilton, 2006),

3. le fait de vouloir exprimer son identité au travers du choix de la cause à qui on lègue (Sargeant et Shang, 2008),

4. le bénéfice des réductions fiscales (Sargeant et Hilton, 2005 ; Sargeant et Shang, 2008 ; Sargeant, Wymer et Hilton, 2006),

5. le prestige de faire un legs à une ONG (Sargeant et Shang, 2008),

6. la réciprocité (Sargeant et Hilton, 2005 ; Sargeant et Shang, 2008 , Sargeant, Wymer et Hilton, 2006),

7. la volonté de limiter l’héritage familial (Sargeant et Shang, 2008).

Ces motivations semblent être de différents niveaux et certaines motivations en précédent d'autres. Par exemple, le fait de ne pas avoir d'enfant explique par la suite la motivation à l'immortalité symbolique. Nous avons donc tenté de structurer les différentes motivations au legs au travers du schéma suivant (figure 1). Chaque élément du schéma sera détaillé par la suite. Nous avons exclu du schéma la motivation d'incitation fiscale car elle n'apparaît qu'aux Etats-Unis où le testateur peut bénéficier de déductions fiscales très tôt du fait d'avoir mis une ONG sur son testament. En France, aucun des collecteurs de fonds rencontré ne pense qu'il existe de motivation fiscale dans le legs. 


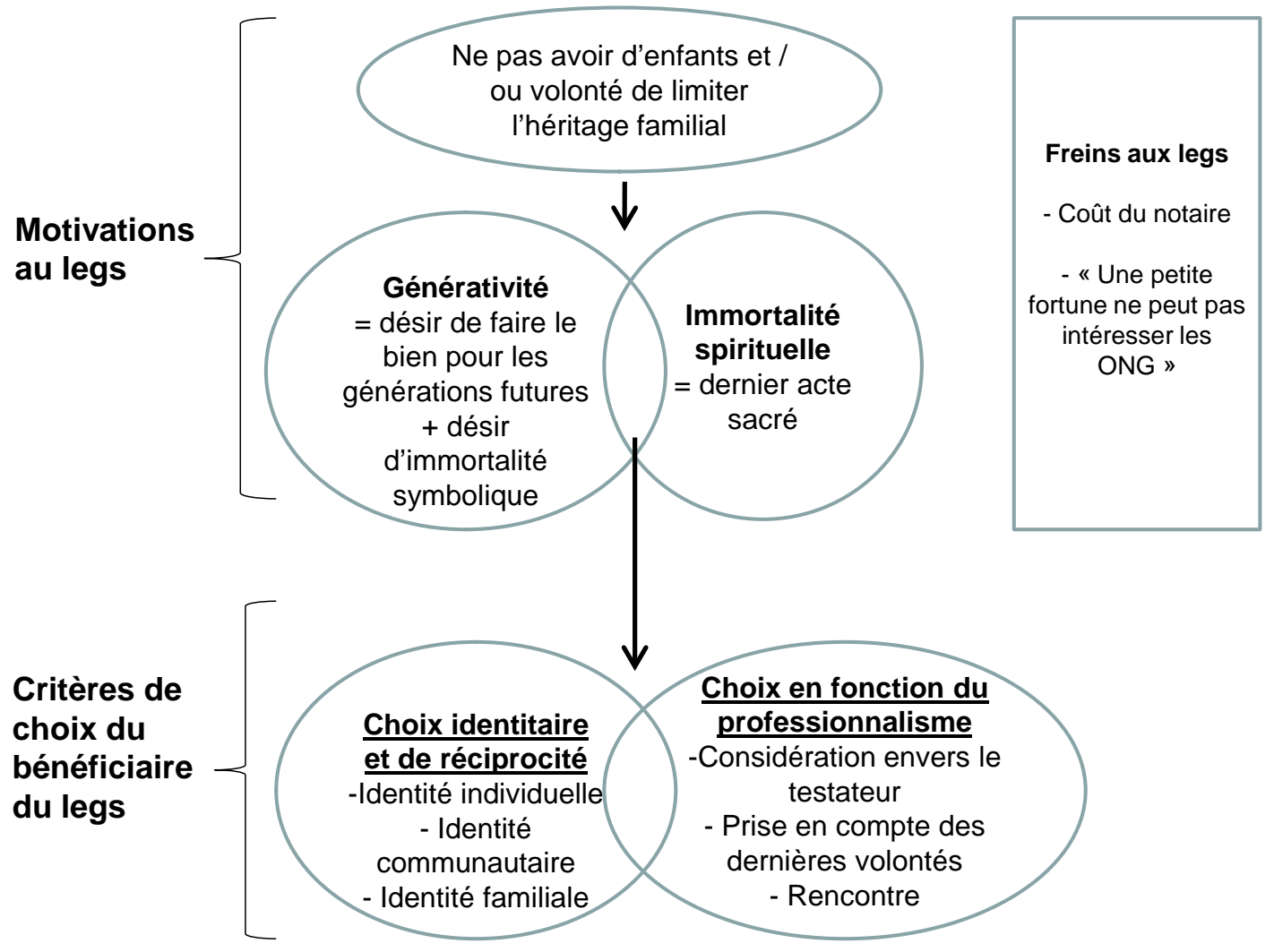

Figure 1 : les freins et les motivations au legs et le choix du bénéficiaire

\section{- $\quad$ L’absence d'enfant : élément déterminant de la collecte de legs}

L’écrasante majorité des testateurs n’ont pas d'enfant. Ainsi, une recherche américaine récente s’est intéressée au profil socio-démographique des testateurs en faveur d'ONG (vs. des testateurs qui n’ont pas mis d’ONG dans leur testament) (James, 2009). Ce chercheur a travaillé sur la base d’un panel de 9244 personnes ayant déjà rédigé leur testament au profit d'associations vs. des testateurs ayant rédigé un testament uniquement au profit de leurs proches. Ses résultats montrent que le facteur le plus explicatif du legs est l'absence d'enfant, suivi par le fait d'être bénévole et de donner plus de 500 dollars par an à des associations. De fait, notre étude qualitative montre qu'en France, les testateurs sont également en très grande majorité sans enfants (entre $89 \%$ et $98 \%$ selon les collecteurs interrogés). Les testateurs avec enfants existent néanmoins et ont deux profils. Soit ils sont très riches et décident en accord avec leurs enfants de ne pas leur donner l'intégralité de leur fortune. Soit ils souhaitent limiter l'héritage d'une progéniture avec laquelle ils sont en conflit. Cette absence d'enfant va expliquer par la suite le comportement du testateur, notamment en termes de générativité. 


\section{- $\quad$ Le choix de léguer guidé par deux grandes motivations}

Au regard de la littérature et de l'analyse de la liste des organisations qui collectent le plus de legs, deux grandes motivations au legs sont à l'origine de ce comportement : (a) le besoin de générativité, (b) la quête d’immortalité spirituelle. Si la générativité apparaissait en filigrane dans la littérature (notamment au travers de l'immortalité symbolique), l'immortalité spirituelle est une variable explicative du legs aux ONG qui apparaît ici lorsqu'on analyse le positionnement des plus grands collecteurs de legs en France. Nous présentons ci-après les deux concepts.

\section{Besoin de générativité}

Le concept de générativité a été introduit il y a 50 ans par Erik Erikson (Erikson, 1963). «Générativité versus stagnation » est la septième des huit étapes de la vie humaine, et dans sa formulation initiale est d'abord associée à l'âge adulte moyen. Le concept de «générativité » recouvre le désir de vouloir assurer une transmission utile pour les générations futures (Erikson, 1963). De façon plus précise, McAdams, Hart et Maruna (1998) présentent une véritable « anatomie de la générativité » et mettent en évidence l'existence primordiale de deux désirs intérieurs : un désir d'immortalité symbolique et un désir d'être nécessaire aux autres - « a need to be needed ».

L’immortalité symbolique fait référence à l'idée d'avoir toujours son nom quelque part après sa mort (Hunter et Rowles, 2005). Ce besoin d’immortalité symbolique n’est pas qu'un besoin lié au nom, nos entretiens montrent en effet que les testateurs ont également une grande angoisse liée à l’oubli de leur histoire personnelle. Léguer revient alors à s'assurer de l'immortalité symbolique de son nom et de son histoire personnelle. Dans la littérature sur la générativité, les chercheurs affirment que le besoin de générativité s’assouvit dans le fait d’avoir des enfants, d'enseigner, de transmettre ses biens ou de pouvoir raconter son histoire (au travers de livres, de journaux intimes etc.). Les testateurs n'ayant pas d'enfants vont donc vouloir exprimer leur désir d'être nécessaire aux autres au travers du legs d’objets spéciaux ${ }^{2}$ (Bergadàa et al., 2011), du legs immobilier ou du legs de leur fortune aux générations futures via le legs aux ONG. L’analyse de la liste des organisations qui collectent le plus de legs en France est d’ailleurs révélatrice de l’importance de la cause de l’enfance (tableau 1). Il semblerait donc qu'il existe un transfert entre le fait de ne pas avoir eu d'enfants et la volonté d'être nécessaire à d'autres enfants.

\footnotetext{
2 Bergadàa et Urien se consacrent aux dons d’objets dits « spéciaux » ou « chéris » : ces objets ont, aux yeux de leur possesseur, une valeur indépendante de l'opportunité d'échange (Richins, 1994). Cette valeur dépend en grande partie de la signification de l'objet pour l'individu. Il peut s'agir par exemple de sa robe de mariée, d'un album photo, d'un bijou sans grande valeur financière mais avec une valeur affective forte.
} 
Tableau 1 : Liste des 24 organisations qui collectent le plus de legs en France (Montant des legs en millions d'euros réalisés) ${ }^{3}$

\begin{tabular}{|c|c|c|c|}
\hline Nom de l'organisation & 2008 & 2009 & 2010 \\
\hline Eglise de France & 80 & 89 & 85 \\
\hline Fondation d'Auteuil & 67 & 71 & 78 \\
\hline Fondation de France & 58 & $613^{4}$ & 46 \\
\hline Ligue Contre le Cancer & 33 & 40 & 37 \\
\hline Institut Pasteur & 41 & 40 & 36 \\
\hline Secours Catholique & 31 & 29 & 31 \\
\hline Fondation pour la Recherche Médicale & 23 & 23 & 24 \\
\hline SPA - Société Protectrice des animaux & 24 & 21 & 21 \\
\hline $\begin{array}{l}\text { ARC - Association pour la Recherche sur le } \\
\text { Cancer }\end{array}$ & 24 & 21 & 19 \\
\hline Petits frères des Pauvres & 24 & 19 & 19 \\
\hline Institut Curie & 19 & 13 & 14 \\
\hline Croix Rouge Française & 11 & 10 & 13 \\
\hline Association des Paralysés de France & 9 & 10 & 12 \\
\hline Unicef France & 5 & 5 & 7 \\
\hline Médecins sans frontières & 4 & 5 & 7 \\
\hline Ordre de Malte & 4 & 5 & 7 \\
\hline SOS village d'enfants & 7 & 4 & 6 \\
\hline Association Valentin Haüy & 8 & 8 & 5 \\
\hline Fondation Abbé Pierre & 7 & 8 & 5 \\
\hline Raoul Follereau & 4 & 5 & 5 \\
\hline Médecins du Monde & 5 & 5 & 4 \\
\hline Secours populaire Français & 4 & 5 & 4 \\
\hline Restos du cœur & 3 & 3 & 4 \\
\hline Armée du salut & 6 & 2 & 2 \\
\hline Total sur ces 24 organisations & $\begin{array}{c}506 \\
\text { millions } \\
\text { d'euros }\end{array}$ & $\begin{array}{c}1054 \\
\text { millions } \\
\text { d'euros }\end{array}$ & $\begin{array}{c}491 \\
\text { millions } \\
\text { d'euros }\end{array}$ \\
\hline
\end{tabular}

Légende : tableau réalisé par les auteurs à partir des rapports d'activité de chaque structure, mis en ligne.

\section{Besoin d'immortalité spirituelle}

Comme le rappellent Ammari et Özçalar-Toulouse dans un chapitre intitulé : «Les racines religieuses de la charité », les trois principales religions valorisent le don aux anonymes. Il est donc du devoir du croyant de faire des dons toute sa vie et de réaliser un don ultime à destination de sa communauté religieuse. Cette motivation fait écho à la dimension spirituelle du legs qui recouvre une dimension sacrée et un désir d'être en lien direct avec l'au-delà. De fait, une étude quantitative réalisée aux Etats-Unis a pu identifier que les testateurs au profit d'ONG sont plus pratiquant que les testateurs au profit unique de leur famille (James, 2009). En France, le tableau 1 témoigne de

\footnotetext{
${ }^{3}$ Les legs réalisés s'entendent comme les sommes entrés dans les comptes de l'année après cession des actifs immobiliers ou autres. Pour des raisons strictement comptables seuls les legs définitivement encaissés figurent au bilan. Les autres sont inscrits dans les produits à recevoir même si 99\% du legs est déjà encaissé.

${ }^{4}$ La Fondation de France a bénéficié en 2009 de deux legs totalement exceptionnels : l'un de 467 millions et l'autre de 94 millions.
} 
l'importance des organisations religieuses dans la collecte de legs ; l'église de France est d'ailleurs la première organisation collectrice de legs. Cette attente d'immortalité spirituelle explique en partie le fait que les institutions religieuses, pour qui le don est lié à la dimension sacrée, représentent une part importante de la collecte de legs du fait de leur légitimité à promettre l’au-delà.

\section{Le choix de l'institution bénéficiaire : une question identitaire et un besoin de professionnalisme}

Une fois que le donateur a pris sa décision de faire un legs pour une organisation extérieure à sa famille, il lui reste à choisir le bénéficiaire de son legs. Par exemple, si la personne décide de léguer pour la recherche sur le cancer, vers quelle association va-telle se tourner ? La Ligue contre le Cancer, l’ARC, l'Institut Curie, la Fondation pour la Recherche Médicale, l'Institut Gustave Roussi et de multiples autres structures vont entrer en concurrence pour être bénéficiaire du legs. Deux grands critères de choix vont alors être utilisés par le testateur: des critères identitaires et des critères liés au professionnalisme perçu de l'organisation.

\section{- Un choix identitaire et de réciprocité}

A l'instar des travaux de recherche d'Aaker et Akutsu (2009), le don est souvent l'expression identitaire de l'individu qui comporte trois dimensions: identité individuelle, identité familiale, identité communautaire. Ces dimensions identitaires sont intimement imbriquées dans un désir de réciprocité envers une cause importante dans le parcours de vie du donateur.

L’identité individuelle fait référence à l'histoire individuelle du donateur et aux organisations importantes dans sa construction identitaire. A ce titre, les établissements d'enseignement supérieurs sont particulièrement bien placés pour recevoir des legs car ils sont des acteurs majeurs de la construction identitaire de l'individu. Par exemple, aux Etats-Unis, il est culturellement admis de rendre à l'institution ce qu'elle nous a donné. De même, le choix de la cause animalière est relative à la place accordée aux animaux dans sa vie personnelle et exprime un remerciement anticipé en échange du fait que l’association s’engage à prendre en charge l'animal domestique du futur défunt.

L’identité familiale fait référence à l'identité de l'individu construite par rapport à ses relations personnelles dans le cercle familial. La mort d'un proche due à un cancer ou à un accident de la route peut induire chez l'individu le souhait de léguer à une 
association spécifique rattachée à cet évènement qui a marqué son histoire familiale. Ainsi, le tableau 1 reflète l'intérêt de la cause médicale dans le choix des testateurs et notamment du cancer (première cause de décès en France avec 150000 morts par an).

L'identité communautaire correspond à la construction identitaire de l'individu en référence à un groupe auquel il appartient ou souhaite appartenir. L’identité communautaire a une forte influence dans les domaines de l'enseignement supérieur et de la culture. Ainsi, le testateur a la sensation que son école, son université ou son musée préféré fait réellement partie intégrante de son identité. Le testateur est alors souvent en attente d'être valorisé de son vivant par ce type d'institution qui lui donne le sentiment d’appartenir à une communauté spécifique.

\section{- Besoin de professionnalisme pour assurer la succession du défunt}

Au-delà du choix identitaire, le testateur fait également son choix à partir du professionnalisme perçu de l’organisation bénéficiaire du legs. Deux auteurs anglosaxons ont montré que les testateurs étaient plus attentifs aux performances de l'association, à la considération qu'elle leur porte et à sa communication que les «non testateurs » (Sargeant et Hilton, 2005). D’après les professionnels que nous avons interrogés, les marques d'attention apportées aux testateurs se cristallisent sur leurs souhaits posthumes et sur la qualité de la rencontre. Nous reviendrons dans la deuxième partie de cet article sur les implications managériales de ces attentes.

Au-delà des motivations à léguer, il y a encore 8 milliards de legs potentiels qui vont à l'Etat faute de démarche de la part du testateur. On peut alors s’interroger sur les freins des testateurs potentiels.

\section{Les freins au legs}

Les freins au legs ont été très peu étudiés dans la littérature. Seuls Sargeant, Hilton et Wymer (2006) ont travaillé sur ce sujet au travers de 8 tables rondes de donateurs. Leurs résultats permettent d'identifier 3 freins : le coût du notaire, le manque de temps et la pensée selon laquelle son legs n'est pas utile pour l'association.

\section{- Le coût du notaire et le manque de temps}

Le coût du notaire est un frein réel aux Etats-Unis où le testateur doit avoir recours à un avocat pour se faire aider dans la rédaction de son testament avant de le faire valider par son notaire. La loi sur les successions est très complexe et nécessite donc une expertise 
extérieure et beaucoup de temps. Ceci n’est pas le cas en France, même si certains collecteurs rencontrés ont soulignés que bon nombre de personnes ne savent pas le prix que coûte le dépôt de son testament et surestiment ce coût.

\section{- Un « petit » legs ne peut pas être utile}

L’idée selon laquelle un petit legs n’intéresse pas les ONG est également un frein au legs qu'il faut arriver à lever dans l'esprit des testateurs. Certains testateurs croient en effet que seules les grandes fortunes intéressent les ONG dans la mesure où ce sont ces legs qui sont très médiatisés.

Comme le montre cette première analyse, les motivations et freins du legs regroupent à la fois des dimensions affective, identitaire et utilitaire du legs. Comment les organisations prennent-elles en compte ces différentes facettes ? Comment le marketing peut-il s’adapter à ce contexte décisionnel unique et paradoxal ?

\section{Les spécificités de la collecte de legs en termes d'offre, de communication et de relation}

Pour répondre à ces questions une étude qualitative a été menée auprès de 21 professionnels de la collecte de legs. Notre recherche révèle que pour s'adapter aux attentes des testateurs potentiels et afin de lever les freins aux legs, les organisations qui récoltent le plus de legs sont celles qui (a) détiennent la plus forte légitimité pour répondre au besoin d'immortalité symbolique et spirituelle (b) communiquent de façon indirecte et implicite sur ce sujet tabou de la mort associée à l'argent, et (c) créent un lien relationnel fort avec le testateur de nature à prouver leur professionnalisme.

\section{La légitimité à offrir l'immortalité symbolique et spirituelle}

Les testateurs décident de léguer leur bien et/ou leur argent dans l'espoir de laisser une trace mais aussi dans l'espoir de bénéficier d'une certaine reconnaissance sociale, c'est ce que l'on a appelé le besoin d’immortalité symbolique. Certains souhaitent également atteindre l'immortalité spirituelle en associant le legs à une dimension sacrée symbolisée par les institutions religieuses. Face à ces attentes, les organisations qui attireront le plus de legs sont celles qui détiendront une légitimité pour répondre à ces besoins d’immortalité. Nous entendons ici par légitimité, la légitimité cognitive (Suchman, 1995), qui suppose qu’une organisation apparaîtra légitime à répondre aux 
besoins d'immortalité si elle incarne par ses valeurs et ses missions des dimensions symboliques ou spirituelles.

\section{- Le besoin d'immortalité symbolique.}

Comme nous l’avons souligné précédemment, les testateurs ont une vraie angoisse face à l'oubli de leur nom. Différents types d’organisations sont légitimes pour mettre en place des dispositifs honorant la mémoire du testateur. En effet, «préserver, restaurer et exposer des cuvres ", "développer des villages pour l'éducation des enfants », «travailler pour la recherche médicale» ou encore "financer des bourses d'étudiants » représentent des missions qui raisonnent en terme de générativité et font sens avec la légitimité à offrir une immortalité symbolique. Ces différentes institutions détiennent des outils de reconnaissance qui reposent, par exemple, sur l'inscription du nom de la personne à côté de l’objet donné, dans la salle rénovée grâce au don, ou encore une plaque commémorative sur un bâtiment. Les établissements d'enseignement supérieur peuvent, eux, offrir des bourses au nom du testateur ou lui dédier une salle à son nom. Au-delà de ces différents exemples, dans la pratique, très peu d'organisations ont mis en place de telles actions alors qu'elles ont une vraie légitimité à offrir de l’immortalité symbolique. En effet, cette immortalité symbolique fait référence au « contre don » offert par l'institution et heurte souvent les conseils d'administrations qui considèrent que le legs doit rester «pur » et ne pas être décidé en fonction d’une promesse d'avoir son nom quelque part pour l'éternité. Pourtant comme nous l'avons montré précédemment cela correspond à une motivation forte du legs chez certains individus.

\section{- Le besoin d'immortalité spirituelle}

Peu d'organisations portent les valeurs du sacré. Seulement quelques unes sont perçues comme légitimes pour permettre au défunt d'atteindre le « paradis ». L’Eglise de France représente l'organisation qui collecte le plus de legs en France car elle incarne par excellence le sacré recherché par les testateurs. Grâce à sa légitimité à apporter l’immortalité spirituelle, le Secours Catholique a 75\% des legs qui lui viennent de personnes non donatrices de l'association (contre 50\% en moyenne pour les autres structures). Ceci montre qu'un nombre important de personnes peuvent léguer à une organisation sans avoir eu de contact préliminaire mais uniquement en fonction de sa réputation et sa légitimité à répondre aux attentes ici spirituelle du défunt. D’ailleurs, le Secours Catholique s'engage dans sa brochure sur cette dimension : "Le Secours Catholique fait célébrer une messe pour vous dès qu'il apprend votre décès ainsi qu'au 
premier anniversaire de votre mort. Si vous l'avez nommé légataire universel, il fera également célébrer 18 messes à vos intentions ».

\section{Une communication spécifique pour parler d'un sujet tabou}

Le legs s'inscrivant autour des questions d'argent et de mort est encore aujourd'hui considéré comme un sujet tabou, difficile à aborder. En effet, le legs n'est pas un acte interdit mais il est difficile de communiquer sur ce sujet avec les personnes concernées, car indéniablement, on associe l'argent à leur mort. Frédéric Grosjean de l'Institut Pasteur observe que « parfois, certaines personnes sont capables de parler de leur mort de manière très directe, sans détournement, mais pour d'autres, c'est difficile. D’ailleurs, ces personnes utilisent beaucoup de métaphores pour qualifier la mort et n’en parlent pas directement ». Ce déni fait référence à la notion d'anxiété face à la mort qui a été définie comme "un ensemble de réactions affectives négatives, d'intensité variable, provoqué par des idées conscientes et non conscientes relatives à la disparition de soi » (Urien et Guiot, 2007). Ce concept comporterait plusieurs composantes telles que, par exemple, l'anxiété relative à l'état physique et mental qui précède immédiatement la mort - c'est à dire l'anxiété relative à l'agonie; l'anxiété relative à la difficulté de concevoir sa propre mort ; ou encore celle correspondant à la peur du corps après la mort. La mort est ainsi devenue, en France, un sujet sur lequel il est difficile de communiquer et les professionnels des obsèques ont d'ailleurs remarqué que les familles ne veulent pas prolonger le contact avec le défunt et cherchent à réduire le temps où elles sont en possession du corps (Cacqueray, 2002). Au final, le legs associant la mort et l'argent est considéré comme un sujet tabou qui restreint la liberté d'en parler en raison des convenances sociales, morales ou religieuses (Sabri O et al., 2010). Le legs en tant que sujet tabou qui associe la mort et l'argent demande une communication à la fois (a) de sensibilisation pour tenter de lever le tabou et (b) d’information afin de répondre aux questions plus pratiques des testateurs potentiels.

\section{- Une démarche de sensibilisation pour lever le tabou}

Le discours implicite détient une place importante, notamment au travers des journaux d'informations. Cette démarche est d'ailleurs adoptée par beaucoup d'associations qui ont une réelle pudeur à réaliser un mailing « spécial legs ». Le journal de l’organisation pour parler du legs s'avère efficace. Ainsi, les résultats d'une étude, menée par Médecins du Monde, ont montré que comparé au mailing et à l'annonce presse avec coupon à renvoyer, c'est «le journal des donateurs » qui assurait le plus de retours. Tiré à 350000 exemplaires, le journal des donateurs a ainsi engendré environ 
700 coupons de demande de brochure dans l'année. L’intérêt du journal de l'organisation qui s’apparente à un « magazine de marque » est qu'il conjugue à la fois un discours pédagogique, interactif et implicite (1). La dimension implicite du « magazine de marque » trouve ici dans le contexte du legs toute sa pertinence. En effet, le discours implicite permet non seulement la pluralité des interprétations d'un même discours selon les besoins des personnes; mais il est aussi l'argument vers lequel on veut amener le destinataire à adhérer, sans avoir à le lui dire (Meyer, 1982). Ainsi, la fondation HEC a fait paraître dans sa lettre de mars 2010, le témoignage d'une jeune femme, qui en expliquant les raisons pour lesquelles son père avait fait un legs à la fondation HEC, sensibilise de façon implicite les plus anciens diplômés de l'école. Ce témoignage permet, d'une part, de présenter le legs comme un moyen de remercier l'institution et reprend l'idée de «transmission » en soulignant que, grâce aux legs, de nouveaux étudiants pourront bénéficier d'une bourse pour suivre une formation à HEC. D’autre part, en faisant témoigner la fille du testateur, cet article peut permettre de déculpabiliser les personnes qui seraient freinées par l’idée de devoir justifier ce choix à leurs proches. Finalement, ce type de communication sur un sujet aussi tabou que le legs, accorde une place majeure à la décision personnelle en laissant l'individu interpréter le message.

\section{- Une campagne d'information sur un sujet mal connu}

Là encore la communication va jouer un rôle important pour informer de façon pédagogique. La brochure dédiée aux legs joue ici un rôle central. Cette brochure rassure la personne sur la pérennité de l’organisation, lui explique ses missions, ses valeurs afin que le testateur puisse identifier l'utilité de son legs. Bien souvent ces brochures utilisent alors des images positives. Ces images pourront renforcer le désir de générativité vu plus haut en mettant en évidence une imagerie liée à la transmission : un enfant avec son grand père, une jeune pousse d'arbre, une main vieille qui serre une main d'enfant etc. Certaines organisations, comme le Secours Catholique, considèrent que les images doivent représenter la finalité du legs (réalisations / bénéficiaires) afin que le testateur puisse se projeter (Destrée, 2009). D’un point de vue plus pragmatique, la brochure est aussi un excellent support pour guider les individus dans leurs démarches administratives. Ainsi certaines brochures de legs donnent des modèles de lettre au cas où la personne souhaite rédiger son testament toute seule et l'envoyer directement à l'association. Cette démarche permet de lever certains freins en évitant ainsi de passer par le notaire et de devoir se justifier. Pour finir, les différents professionnels sont unanimes pour affirmer qu'outre son rôle informationnel la brochure est l'outil qui va 
permettre le premier contact avec le testateur potentiel, il est donc essentiel de personnifier l'organisation. Dans la plupart des brochures de legs, on peut donc trouver la photo de la personne en charge des legs dans l'organisation ainsi que ses coordonnées. Ces dernières informations constituent un moyen très efficace pour rentrer en relation et débuter une étape essentielle de la collecte de legs : la relation privilégiée et personnelle avec le testateur potentiel.

\section{Spécificité relationnelle : la recherche de l'attachement émotionnel et de}

\section{la confiance pour renforcer le professionnalisme perçu de l'association}

Comme nous l'avons vu dans la première partie de cet article, le choix de l'ONG bénéficiaire du legs se fait également en fonction de la quête identitaire du donateur et du professionnalisme perçu de l'organisation. Nos entretiens permettent de montrer que la politique relationnelle va influencer ces perceptions si elle se base sur la confiance et l'attachement émotionnel. Comme le souligne un responsable d'une agence conseil, on ne doit pas oublier que le testateur est une personne en «souffrance affective ». Dans 9 cas sur 10 le testateur est une personne qui n'a pas eu d'enfants, ce qui, en fin de vie, est vécu de manière négative. Il est donc essentiel de les rencontrer pour nouer une relation personnalisée avec eux. On peut ainsi citer deux collecteurs de fonds : «Les testateurs ont une urgence à raconter leur vie, à transmettre à quelqu'un leur existence. Ils sortent les albums photos, expliquent leur vie en image » (Adfinitas); en d'autres termes « quelque part, dans le legs, on reçoit la vie de quelqu'un » (Ernst et Young).

Avec ce premier contact et au fil des rencontres, la relation nouée avec le testateur amène la confiance, l'attachement et la reconnaissance d'un certain professionnalisme. C'est lorsque la confiance se transforme en attachement que l'organisation se verra choisie par le testateur. On peut relater ici le cas d'une personne âgée vivant seule et sans famille qui est entrée en contact avec la Croix Rouge dans le but de se renseigner sur les legs. Lors de ses quelques rencontres avec la personne en charge des legs, la donatrice a eu des échanges de grande qualité avec celle-ci. Alors que cette personne âgée pensait faire un legs mineur à la Croix Rouge et léguer sa maison à une autre association à laquelle elle était une donatrice fidèle, elle a finalement inversé sa décision. L'analyse de ce cas montre que l'attachement que l'organisation a su créer avec le testateur potentiel est un des points clés de la collecte de legs.

Plus particulièrement, pour créer ces liens attachants (Thomson, MacInnis et Park, 2005) l'association va chercher d'une part a) à répondre aux besoins identitaires des individus (cf. motivations) et va chercher d'autre part b) à avoir des marques 
d'attention. Ces actions ne s’arrêtent pas au moment où la personne a pris sa décision, la grande majorité des organisations poursuivent la relation avec les testateurs même après qu'ils aient signé leur testament en leur faveur.

- $\quad$ La réponse aux besoins identitaires se traduit par exemple par des invitations aux expositions et évènements liés à l'organisation. Le Louvre a, par exemple, créé plusieurs cercles de donateurs, qui se rencontrent au travers de visites privées et d'un gala annuel. Il en est de même dans les organisations culturelles aux Etats-Unis qui n’hésitent pas à créer des cercles de futurs testateurs. Selon la responsable de la collecte au China Institute de New York : «la personne doit se sentir privilégiée, unique». Dans le domaine des organisations de solidarité, seuls l'Institut Pasteur et Greenpeace réalisent ce type d'invitations pour leurs testateurs.

- En revanche, les autres organisations de solidarité vont se concentrer sur différentes «marques d'attention" comme les envois de vœux pour Noël, l'anniversaire, prennent aussi des nouvelles, informent de l'actualité de l'organisation, et surtout, répondent à toutes les interrogations que peuvent se poser les testateurs. Dans ce cadre, tous les petits détails ont leur importance. Face à une population âgée les courriers manuscrits sont de rigueur. C'est le signe d'une attention particulière, d'une relation privilégiée grâce à la perception d'un traitement de faveur que les organisations veulent offrir aux testateurs même après leur mort (Butori, 2010). A titre anecdotique, le Secours Catholique a un jour reçu une pétition des habitants d'une maison de retraite qui trouvaient scandaleux que l'association n'ait pas mis une plaque de remerciement sur la tombe d'un pensionnaire ayant légué à l'association, bien entendu, l'association s’est exécutée.

A l'heure où on parle énormément de «marketing relationnel », la stratégie de legs semble une illustration parfaite de ce marketing puisqu'elle repose sur la connaissance intime de l'individu et l'adaptation totale aux volontés de ce dernier. Comme le dit Donna Thomas : «fundraising is relationship. If you don't like to cultivate relationships with others you don't know, you mustn't do this job $»^{5}$. De fait, quelle organisation peut prétendre aujourd'hui connaître toute la vie de l'individu, ses relations familiales, ses angoisses, ses émotions, ses valeurs, le nom de son animal domestique, les objets auxquels il tient particulièrement et ses dernières volontés ?

Les attentions particulières avant et après la mort du testateur permettent de créer à la fois un attachement avec le testateur mais aussi de créer une réputation positive et de

\footnotetext{
${ }^{5}$ Traduction : « la collecte de fonds repose sur le relationnel. Si vous n’aimez pas entretenir des relations avec les personnes que vous ne connaissez pas, vous ne devez pas occuper cet emploi ».
} 
professionnalisme auprès de l'entourage du bénéficiaire. Il est rassurant pour le testateur de voir que l'organisation s'engage à respecter ses volontés et à prendre soin des biens auxquels il tient. A ce sujet, les petits frères des Pauvres sont certainement les plus professionnels de la relation avec le testateur (encadré 3).

\section{Encadré 3 - Les petits frères des Pauvres : un exemple d'association professionnelle dans l’observation des dernières volontés des testateurs}

Les petits frères des Pauvres ont une grande tradition de collecte de legs car cette source de financement fait partie de leur histoire. C'est la seule association, à notre connaissance, à avoir mis en place un système d'appel 24 h sur 24,7 jours sur 7 , qui permet aux proches du défunt d'appeler l'association dès que la personne vient de décéder. Ceci permet à l'association de pouvoir s'assurer que les dernières volontés de la personne seront réellement respectées et de prendre en charge le dossier de succession immédiatement. De plus, les petits frères des Pauvres ont investi dans un local qui leur permet de stocker les biens des personnes en attente de la clôture de la succession : "ça nous permet par exemple, si une personne part à la maison de retraite, de pouvoir prendre les meubles qu'elle ne peut pas emporter, de les mettre dans un container, puis, si elle veut les récupérer, de les lui redonner; si elle meurt, et que la donation nous est destinée, les meubles sont déjà en notre possession ». Ceci leur permet également de vendre les objets donnés ou légués à leur juste valeur car l'association peut alors organiser des ventes thématiques aux enchères qui ont plus d'impact en termes de ventes. Ceci est important aux yeux du testateur qui souhaite que ses biens ne soient pas «bradés ». Les petits frères des Pauvres s'occupent aussi de tout le processus des obsèques, même au-delà, du choix de la concession au fleurissement annuel de la sépulture, ce qui leur donne une image à la fois très humaine et très professionnelle qui leur permet de se différencier des autres associations qui en général n’assurent ni les obsèques ni l'entretien de la sépulture.

\section{Un investissement financier important pour garantir le professionnalisme}

\section{de la collecte de legs}

Au final, les spécificités de la collecte de legs en termes d’offre, de communication et de relation supposent un investissement financier important pour l'organisation qui doit mettre en place un service dédié à ces opérations. Médecins du Monde investit aujourd'hui, 450000 euros par an pour collecter 5 millions d'euros de legs. Il en est de même pour le Secours Catholique qui dépense 350000 euros par an (sans les salaires) pour collecter 28 millions d'euros de legs. L’Institut Pasteur dépense 279000 euros de communication par an pour collecter entre 40 et 80 millions par an. Toutefois malgré ces exemples, la majorité des associations n’ont pas engagé une stratégie de collecte de legs pour deux raisons principales. La première concerne la dimension long-terme de l'investissement qui freine les organisations qui doivent attendre 5 à 7 ans pour bénéficier financièrement des premiers legs. La deuxième raison concerne les questions 
éthiques soulevées par le legs. Pour palier ces réticences déontologiques, la pratique de la collecte de legs se fait généralement dans le respect d'une charte éthique propre à toute organisation pour s'assurer de ne pas s'exposer à un risque de captation d'héritage. Enfin, nos entretiens ont fait émerger le tabou du sujet du legs pour certains conseils d'administration peu à l'aise avec ce mode de collecte : « ca ne fait que deux ans qu'on a commencé, la collecte de legs et les gens en interne ont l'impression qu'on pousse les gens vers la mort pour récupérer leur argent »(Greenpeace).

\section{Conclusion}

Cet article apporte une contribution à la littérature sur le legs au travers de l'analyse des pratiques des professionnels de la collecte dans trois secteurs distincts. En France, force est de constater que la collecte de legs est très peu professionnalisée dans les secteurs de la culture et de l'enseignement supérieur. Au terme de cette recherche, nous pouvons conclure qu'hormis de grandes institutions, la plupart des organisations n’ont pas encore développé de démarches professionnelles pour la collecte de legs. Pourtant les professionnels qui s'y sont engagés affirment que le ratio collecte de legs / fonds affectés est très bon et nettement supérieur à la collecte de dons. Les pratiques outre Atlantique montrent l'importance du contexte culturel dans la collecte de legs (annexe 2). Une meilleure connaissance et une meilleure information pour modifier la représentation sociale du legs, semble donc représenter la clé de l'évolution de la collecte de legs au sein des organisations.

Cet article a également insisté sur les implications managériales liées au processus de décision du legs en mettant en évidence le rôle fondamental de la communication et du marketing relationnel. Face à des attentes bien particulières, la collecte de legs se fonde essentiellement sur :

- la légitimité à offrir l’immortalité symbolique et spirituelle,

- l'utilisation d'une communication informative et implicite face à un sujet tabou,

- la construction d'une relation de confiance et d'attachement sur la base d'un professionnalisme en termes relationnel et de gestion post-mortem.

Le tableau 2 ci-dessous récapitule l’ensemble de ces implications managériales.

\begin{tabular}{|l|l|l|l|}
\hline $\begin{array}{l}\text { Freins et } \\
\text { motivations au legs }\end{array}$ & $\begin{array}{l}\text { Implications } \\
\text { managériales en } \\
\text { termes de légitimité }\end{array}$ & $\begin{array}{l}\text { Implications } \\
\text { managériales en termes } \\
\text { de communication }\end{array}$ & $\begin{array}{l}\text { Implications } \\
\text { managériales en } \\
\text { termes de marketing } \\
\text { relationnel }\end{array}$ \\
\hline $\begin{array}{l}\text { Ne pas avoir } \\
\text { d'enfants et / ou } \\
\text { vouloir limiter } \\
\text { l'héritage familial }\end{array}$ & & $\begin{array}{l}\text { Sensibiliser les donateurs } \\
\text { de l'ONG à la possibilité } \\
\text { de faire un legs via le } \\
\text { journal de l'ONG }\end{array}$ & $\begin{array}{l}\text { Répondre à la } \\
\text { problématique de } \\
\text { " souffrance affective » } \\
\text { en créant une relation }\end{array}$ \\
\hline
\end{tabular}




\begin{tabular}{|c|c|c|c|}
\hline & & $\begin{array}{l}\text { Répondre aux } \\
\text { interrogations des } \\
\text { testateurs sur les } \\
\text { problèmes de succession et } \\
\text { de testament via la } \\
\text { brochure «legs » et le } \\
\text { service juridique de } \\
\text { l'association. }\end{array}$ & $\begin{array}{l}\text { personnalisée et } \\
\text { privilégiée entre le } \\
\text { testateur et le « chargé } \\
\text { de legs ». }\end{array}$ \\
\hline $\begin{array}{l}\text { Générativité : } \\
\text { - Désir de faire le } \\
\text { bien pour les } \\
\text { générations futures } \\
\text { - Désir } \\
\text { d’immortalité } \\
\text { symbolique }\end{array}$ & $\begin{array}{l}\text { Montrer la capacité de } \\
\text { l'organisation à mener } \\
\text { des actions qui profitent } \\
\text { aux générations futures } \\
\\
\text { Proposer des opérations } \\
\text { qui assurent } \\
\text { l'immortalité } \\
\text { symbolique (plaques au } \\
\text { nom du testateur, nom } \\
\text { donné à une bourse } \\
\text { d'étude, à une salle de } \\
\text { classe, à une fondation } \\
\text { etc.) }\end{array}$ & $\begin{array}{l}\text { Rassurer le testateur sur la } \\
\text { pérennité de l’ONG via la } \\
\text { brochure « legs ». } \\
\text { Mettre en avant le lien } \\
\text { intergénérationnel dans la } \\
\text { brochure « legs » (photos, } \\
\text { symboles, etc.). }\end{array}$ & \\
\hline $\begin{array}{l}\text { Désir d'immortalité } \\
\text { spirituelle }\end{array}$ & $\begin{array}{l}\text { Mettre en place des } \\
\text { actions qui offrent une } \\
\text { immortalité spirituelle } \\
\text { (ex : messes offertes en } \\
\text { l'honneur du défunt) }\end{array}$ & $\begin{array}{l}\text { Communiquer sur les } \\
\text { fondements religieux de } \\
\text { l'organisation (si } \\
\text { pertinent) }\end{array}$ & $\begin{array}{l}\text { Développer la capacité } \\
\text { du « chargé de legs » à } \\
\text { échanger sur le plan } \\
\text { religieux avec le } \\
\text { testateur. }\end{array}$ \\
\hline $\begin{array}{l}\text { Choix identitaire et } \\
\text { de réciprocité }\end{array}$ & & $\begin{array}{l}\text { Développer la collecte de } \\
\text { legs auprès des donateurs } \\
\text { qui par essence sont } \\
\text { attachés à l'organisation. } \\
\\
\text { Pour plus de transparence, } \\
\text { informer prioritairement le } \\
\text { futur testateur de toutes les } \\
\text { évolutions de } \\
\text { l'organisation (ex : rapport } \\
\text { d'activité annuel etc.) }\end{array}$ & $\begin{array}{l}\text { Travailler sur les } \\
\text { besoins identitaires de } \\
\text { l'individu lors de sa } \\
\text { rencontre avec le chargé } \\
\text { de legs. } \\
\text { Développer des outils de } \\
\text { valorisation du futur } \\
\text { testateur basés sur } \\
\text { l'identité (ex : invitation } \\
\text { à des soirées au sein de } \\
\text { l'ONG, création de } \\
\text { « cercles de testateurs ", } \\
\text { etc.). }\end{array}$ \\
\hline $\begin{array}{l}\text { Choix en fonction du } \\
\text { professionnalisme de } \\
\text { l’ONG : } \\
\text { - Considération } \\
\text { envers le donateur } \\
\text { - Rencontre } \\
\text { - Prise en compte de } \\
\text { ses dernières } \\
\text { volontés }\end{array}$ & $\begin{array}{l}\text { Pouvoir assurer au } \\
\text { testateur le respect de } \\
\text { ses dernières volontés et } \\
\text { l'organisation de ses } \\
\text { obsèques. }\end{array}$ & & $\begin{array}{l}\text { Assurer la disponibilité } \\
\text { du « chargé de legs » } \\
\text { pour répondre aux } \\
\text { sollicitations des futurs } \\
\text { testateurs. } \\
\text { Entretenir la relation } \\
\text { avec le testateur au fil } \\
\text { du temps (ex : vœux, } \\
\text { carte d’anniversaire, } \\
\text { contact téléphonique } \\
\text { annuel, répondre à ses } \\
\text { demandes de rencontres, } \\
\text { etc.) }\end{array}$ \\
\hline Coût du notaire & & $\begin{array}{l}\text { Informer du coût moyen } \\
\text { d'un dépôt de testament } \\
\text { chez le notaire. } \\
\text { Proposer un } \\
\text { accompagnement juridique } \\
\text { pour assurer la validité du } \\
\text { testament. }\end{array}$ & $\begin{array}{l}\text { Favoriser la prise de } \\
\text { contact avec le } \\
\text { responsable juridique de } \\
\text { l'ONG (photo, contact, } \\
\text { etc.) }\end{array}$ \\
\hline
\end{tabular}




\begin{tabular}{|l|l|l|l|}
\hline & & & \\
\hline « une petite fortune & & $\begin{array}{l}\text { Dans la brochure « legs », } \\
\text { présenter des témoignages } \\
\text { intéresser les ONG » }\end{array}$ & $\begin{array}{l}\text { Le chargé de legs doit } \\
\text { rassurer le testateur sur }\end{array}$ \\
& & $\begin{array}{l}\text { que le legs ne concerne pas } \\
\text { uniquement les grandes } \\
\text { fortunes. }\end{array}$ & \\
\hline
\end{tabular}

Tableau 2 - Implications managériales pour les organisations collectrices de legs

Enfin, cet article est un premier pas vers une meilleure connaissance de la collecte de legs et d'autres recherches pourraient être envisagées sur ce thème. Ainsi, deux voies de recherches nous paraissent particulièrement intéressantes. D’une part, il serait sûrement très riche de pouvoir participer aux rencontres «testateur - chargé de legs » qui ont lieues le plus souvent au domicile de la personne. Ces entretiens durent généralement entre une demi-journée et une journée. Une observation non participante dans une démarche de type interprétativiste pourrait permettre de mieux identifier les freins et les motivations au legs.

Une autre recherche serait intéressante à mener sur les freins et les motivations au legs selon le type d'ONG. Ainsi, la littérature sur le legs s'est essentiellement focalisée sur le legs aux associations humanitaires et nous ne savons quasiment rien sur le legs aux établissements d'enseignement supérieurs ou aux organisations culturelles. Si le legs aux associations humanitaires semble immédiatement utile pour la cause, l’utilisation pour les deux autres types d'organisations est plus abstraite et son effet semble être à plus long terme. Il serait donc intéressant de chercher plus avant sur les ressemblances et les différences de processus de décisions du legs entre les trois types d'ONG : associations humanitaires, établissements d'enseignement supérieurs et organisations culturelles.

\section{Références bibliographiques}

Aaker J.L. et Akutsu S. (2009), Why do People Give? The Role of Identiy in Giving, Journal of Consumer Psychology, 19, 267-270.

Ammari R. et Özçalar-Toulouse N. (2011), « Les racines religieuses de la charité » in Marine Le Gall, Michèle Bergadaà et Bertrand Urien, Don et pratiques caritatives, De Boeck, 51-66.

Bergadàa M., Le Gall Ely M. et Urien B. (2011), Don et pratiques caritatives, De Boeck, 202 pages.

Butori R. (2010), Pour une meilleure utilisation du traitement de faveur, une approche individuelle, Recherche et Applications en Marketing, 25, 1, 33-57. 
Cacqueray (de) C. (2002), La mort confisquée - Essai sur le déclin des rites funéraires, éditions CLD, 143 pages.

Destrée J.M. (2009), Bâtir une stratégie legs - Petits et grands secrets, Actes du 8eme séminaire Francophone de la collecte de fonds, 70 pages.

Dutheil P. H. et Vialle C. (2009), Les libéralités : un potentiel sous exploité ?, Rapport Ernst et Young, 25 pages.

Erikson E. (1963), Childhood and society, 2e éd., New, York, Norton.

Hunter E.G. et Rowles G.D. (2005), Leaving a Legacy: Toward a Typology, Journal of Aging Studies, 327-347.

James R. N. (2009), Health, Wealth and Charitable Estate Planning, Nonprofit and Voluntary Sector Quarterly, 38, 6, 1026-1043.

McAdams, Hart et Maruna (1998), The anatomy of generativity. In D. P. McAdams \& E. de St. Aubin (Eds.), Generativity and adult development. Washington, DC: American Psychological Association.

Mauss M. (1954), The Gift : forms and functions of exchange in archaic societies, London : Routledge and Kegan Paul.

Meyer M. (1982), Logique, langage et argumentation, Paris, Hachette Université.

Michel G. et Vergne J.F. (2000), Un outil de communication pour la distribution : Le magazine de consommateurs, Décisions Marketing, 19, 53-60.

Richardson D. et Chapman G. (2005), What some recent research tells us about planned giving (legacy marketing) in North America, International Journal of Nonprofit Voluntary Sector Marketing, 10, 33-41.

Sabri O, Manceau D. et Pras B. (2010), Le tabou, un concept peu exploité en marketing, Recherche et Applications en Marketing, 25, 1, 59-86.

Sargeant A. et Hilton T. (2005), The final gift : targeting the potential charity legator, International Journal of Nonprofit and Voluntary Sector Marketing, 10, 3-16.

Sargeant A., Hilton T. and Wymer W. (2006), Bequest Motives and Barriers to Giving The Case of Direct Mail Donors, Nonprofit Management and leadership, 17, 1, 4966.

Sargeant A. et Shang J. (2008), Identification, Death and Bequest Giving, Association of Fundraising Professionals, Reports on Fundraising And Philanthropy, 68 pages.

Sargeant A., Wymer W. et Hilton T. (2006), Marketing Bequest Club Membership: An Exploratory Study of Legacy Pledgers, Nonprofit and Voluntary Sector Quarterly, 35, 3, 384-404.

Suchman M.C. (1995), Managing Legitimacy : Strategic and Institutional Approaches, Academy of Management Review, Vol.20, n³, p.571-610. 
Thomson M. MacInnis D. et Park C.W ; (2005), Les liens attachants : mesurer la force de l'attachement émotionnel des consommateurs à la marque, Recherche et Applications en Marketing, 20,1, 79-98.

Urien B. et Guiot D. (2007), Attitude face à la mort et comportement des consommateurs âgés: vers l'élaboration d’une réponse marketing, Décisions Marketing, avril - juin, 46, 23-35.

\section{Annexe 1 - Guide d'entretien}

Parlez-moi de la collecte de legs dans votre organisation ...

Quelles ressources faut-il avoir pour collecter des legs ? (en argent, en hommes)

Qui sont les testateurs potentiels (âges, caractéristiques socio-démographiques) ?

Comment trouver les testateurs potentiels ? Combien viennent du fichier ?

Comment démarcher un testateur?

A partir de quel moment vous déplacez vous chez le testateur?

Comment le convaincre?

Comment entretenir le lien jusqu’à la réalisation du legs ?

Quels sont les risques de la collecte de legs ?

Vous est-il arrivé de refuser des legs ? Pourquoi ?

Avez-vous déjà fait des études sur le comportement des testateurs ? Si oui, lesquelles ?

Quelle autre association / établissement d'enseignement supérieur / établissement culturel faut-il rencontrer sur les legs ?

\section{Annexe 2 - La collecte de legs en Amérique du Nord}

Outre Atlantique, la collecte de legs est différente. Ainsi, la législation n’impose pas les mêmes contraintes vis-à-vis de ses héritiers : un couple avec enfant peut décider de léguer $75 \%$ de sa fortune à une association et $25 \%$ à ses enfants. De plus, le legs est totalement ancré dans la culture américaine, très orientée vers le don. Il n’est pas choquant qu'une personne avec des descendants fasse un legs d'une partie de sa fortune. Ceci nous a également été confirmé par Donna Thomas, collectrice du China Institute de New York. Pour elle, les personnes qui font des legs ont souvent beaucoup d'argent. Elles assurent donc l'avenir de leurs enfants, puis, donnent une partie de leur argent à une organisation. Les individus rédigent également leur testament beaucoup plus tôt qu'en France : 49 ans aux Etats-Unis (Richardson et Chapman, 2005) contre 70 ans en France. 
Selon Emmanuelle Poupeau, ancienne membre du service de collecte du Lycée Français de New York, les américains n’ont aucune réticence à parler de legs : pour eux, ni l'argent, ni la mort ne sont des tabous. D’ailleurs «le programme de collecte de «University South Carolina» sollicite des étudiants pour faire la collecte de legs auprès des anciens. Ils ont même des challenges pour valoriser l'étudiant qui récoltera le plus de promesses de legs ». Il faut également garder en mémoire qu'aux Etats-Unis, d'énormes moyens sont mis sur la collecte de fonds. Ainsi, Arthur Moore souligne : «Havard embauche plus de 200 personnes pour sa collecte. Pour les legs, ils sélectionnent 150 anciens étudiants qui ont déclaré vouloir faire un legs à l'université et n'hésitent pas aller les rencontrer une fois par an, chez eux. Ils les invitent à sortir, à boire le thé, à aller manger au restaurant, on appelle ça « to cultivate the relation » ". 